\title{
Theoretical Prediction and Spectroscopic Fingerprints of an Orbital Transition in $\mathrm{CeCu2Si2}$
}

\author{
L.V. Pourovskii, P. Hansmann, M. Ferrero and A. Georges
}

\section{Linköping University Post Print}

\section{Tweet}

N.B.: When citing this work, cite the original article.

Original Publication:

L.V. Pourovskii, P. Hansmann, M. Ferrero and A. Georges, Theoretical Prediction and Spectroscopic Fingerprints of an Orbital Transition in $\mathrm{CeCu} 2 \mathrm{Si} 2,2014$, Physical Review Letters, (112), 10, 106407.

http://dx.doi.org/10.1103/PhysRevLett.112.106407

Copyright: American Physical Society

http://www.aps.org/

Postprint available at: Linköping University Electronic Press

http://urn.kb.se/resolve?urn=urn:nbn:se:liu:diva-106021 


\title{
Theoretical Prediction and Spectroscopic Fingerprints of an Orbital Transition in $\mathrm{CeCu}_{2} \mathrm{Si}_{2}$
}

\author{
L. V. Pourovskii, ${ }^{1,2}$ P. Hansmann, ${ }^{1}$ M. Ferrero, ${ }^{1}$ and A. Georges ${ }^{1,3,4}$ \\ ${ }^{1}$ Centre de Physique Théorique, CNRS, École Polytechnique, 91128 Palaiseau, France \\ ${ }^{2}$ Swedish e-science Research Centre (SeRC), Department of Physics, Chemistry and Biology (IFM), \\ Linköping University, SE-58183 Linköping, Sweden \\ ${ }^{3}$ Collège de France, 11 place Marcelin Berthelot, 75005 Paris, France \\ ${ }^{4}$ DPMC, Université de Genève, 24 quai Ernest Ansermet, CH-1211 Genève, Switzerland
}

(Received 22 May 2013; revised manuscript received 22 October 2013; published 13 March 2014)

We show that the heavy-fermion compound $\mathrm{CeCu}_{2} \mathrm{Si}_{2}$ undergoes a transition between two regimes dominated by different crystal-field states. At low pressure $P$ and low temperature $T$ the $\mathrm{Ce} 4 f$ electron resides in the atomic crystal-field ground state, while at high $P$ or $T$, the electron occupancy and spectral weight is transferred to an excited crystal-field level that hybridizes more strongly with itinerant states. These findings result from first-principles dynamical-mean-field-theory calculations. We predict experimental signatures of this orbital transition in x-ray spectroscopy. The corresponding fluctuations may be responsible for the second high-pressure superconducting dome observed in this and similar materials.

PACS numbers: 71.27.+a, 71.30.+h, 78.70.Ck, 78.70.Dm

$\mathrm{CeCu}_{2} \mathrm{Si}_{2}$, the first discovered heavy-fermion superconductor [1], still generates a lot of interest due to the peculiar shape of the superconducting (SC) region in its pressure-temperature $(P-T)$ phase diagram. Superconductivity in this compound is observed in a wide range of pressures from 0 to $7 \mathrm{GPa}$ with the $\mathrm{SC}$ critical temperature $T_{c}$ featuring two maxima: $T_{c} \approx 0.6 \mathrm{~K}$ at $P_{c}=0.45 \mathrm{GPa}$, and $T_{c} \approx 2 \mathrm{~K}$ at $P_{c}^{*} \approx 4.5 \mathrm{GPa}$ [2-5]. This double-dome shape of the SC region has also been observed in isoelectronic $\mathrm{CeCu}_{2} \mathrm{Ge}_{2}[6]$ and differs from the $\mathrm{SC}$ phases in other Ce 122-type compounds $\left(\mathrm{CePd}_{2} \mathrm{Si}_{2}\right.$ [7], $\mathrm{CeRh}_{2} \mathrm{Si}_{2}$ [8]), which exhibit a single-dome $\mathrm{SC}$ phase in a much narrower range of pressures around an antiferromagnetic (AFM) quantum critical point. By substituting $10 \%$ of $\mathrm{Si}$ with Ge one may completely separate the two SC domes in $\mathrm{CeCu}_{2} \mathrm{Si}_{2}$ [9], thus suggesting that the $\mathrm{SC}$ domain in pure $\mathrm{CeCu}_{2} \mathrm{Si}_{2}$ is actually a merge of two SC phases with different origins.

The maximum of the low-pressure SC dome has been unambiguously related to an AFM quantum critical point located at $P_{c}$. Indeed, specific heat measurements under small applied pressures in an external magnetic field [10] reveal that small deviations from the nominal stoichiometry stabilize either the AFM or SC phases at zero pressure [11]. The SC transition is accompanied by a lowering of the magnetic exchange energy [12]. It is widely accepted, based on these observations, that the low-pressure SC phase is due to spin-fluctuation mediated pairing, similar to the single-dome $\mathrm{SC}$ in $\mathrm{CePd}_{2} \mathrm{Si}_{2}$ and $\mathrm{CeRh}_{2} \mathrm{Si}_{2}$.

In contrast, no consensual picture has emerged to date for the pairing mechanism in the high-pressure SC phase. The AFM order is already suppressed at pressures significantly below $P_{c}^{*}$, ruling out spin-fluctuation driven SC.
For $P \gtrsim P_{c}^{*}$, the effective mass estimated from the ac specific heat is significantly reduced [13]. The normalstate resistivity around $P_{c}^{*}$ is described by $\rho=\rho_{0}+A T^{n}$, with a large enhancement of $\rho_{0}$ and a non-Fermi liquid exponent $n \approx 1$ [14]. Recent multiprobe transport measurements clearly revealed the proximity of a critical point close to $P_{c}^{*}[4,5]$. It has been proposed [15] that $P_{c}^{*}$ is associated with the critical end point of a first-order valence transition (VT), and that the associated critical fluctuations may provide the pairing mechanism in the high-pressure SC phase [13]. Such a VT, at which the Ce- $4 f$ orbital occupancy $n_{f}$ jumps discontinuously, has been obtained within a single-band periodic Anderson model (PAM) in which an additional repulsion between the conduction electron band and the $f$ orbital is introduced [16]. However, recent x-ray absorption measurements in a wide pressure range from 0 to $7.8 \mathrm{GPa}$ detected only a smooth and weak decrease of $n_{f}$ as a function of pressure, without any marked feature around $P_{c}^{*}$ [17]. These results are in clear contradiction to the proposed valence transition and valence-fluctuation mechanism for SC.

In this Letter, we provide theoretical evidence that $P_{c}^{*}$ is actually associated with an orbital transition between two different crystal-field levels. This conclusion is reached by performing first-principles calculations of $\mathrm{CeCu}_{2} \mathrm{Si}_{2}$ which combine electronic structure methods [density functional theory in the local density approximation (LDA)] with a many-body treatment of the strong correlations in the $\mathrm{Ce} 4 f$ shell [dynamical mean-field theory (DMFT)]. We investigated the evolution of the electronic structure of the normal paramagnetic state as a function of applied pressure and temperature in the range $0<P<8 \mathrm{GPa}$, $7<T<58 \mathrm{~K}$. Our calculations reveal that while $n_{f}$ 
remains close to unity within the whole range, the occupancies of different crystal-field (CF) levels within the Ce $4 f^{1}$ multiplet change drastically as a function of $P$ and/or $T$. At low pressure and temperature the $4 f$ electron is mostly located at the ground-state level of the atomic Hamiltonian, while with increasing $P$ (and $T$ ) the electron weight is transferred to an excited level, which hybridizes more strongly with itinerant bands. The transition as a function of pressure becomes more drastic at low temperature, hinting at a quantum critical point at $P \approx 2.7 \mathrm{GPa}$, in rather close proximity to the maximum of the second SC dome. We show that the low-energy electronic structure is affected by this orbital transition, with the main Kondo resonance changing its orbital character. Finally, we predict distinctive signatures of this orbital transition in nonresonant inelastic x-ray scattering (NIXS) experiments.

We use a fully self-consistent in the charge density LDA + DMFT method [18,19] which combines a fullpotential band-structure technique [20] with the DMFT [21] treatment of the on-site Coulomb repulsion between Ce $4 f$ states. The DMFT quantum impurity problem was solved with the numerically exact hybridization-expansion continuous-time quantum Monte Carlo (CT-QMC) method [22], as implemented in the TRIQS [23] package [24].

We calculated $\mathrm{CeCu}_{2} \mathrm{Si}_{2}$ in its experimental bodycentered tetragonal $\mathrm{ThCr}_{2} \mathrm{Si}_{2}$-type structure (Fig. 1) and at the measured values of the lattice parameters versus $P$ reported in Refs. $[25,26]$. In a tetragonal crystal field the ${ }^{2} F_{5 / 2}$ ground-state multiplet of the $\mathrm{Ce}^{3+}$ ion is split into three doublets: $|0\rangle=a| \pm 5 / 2\rangle+\sqrt{1-a^{2}}|\mp 3 / 2\rangle$, $|1\rangle=| \pm 1 / 2\rangle$, and $|2\rangle=\sqrt{1-a^{2}}| \pm 5 / 2\rangle-a|\mp 3 / 2\rangle$. As one sees in Figs. 1(a) and 1(b), the CF states $|0\rangle$ and $|2\rangle$ differ by their orientation in the (001) plane: while the lobes of the $|2\rangle$ point along [110] towards the nearest neighbor $\mathrm{Si}$ sites, the lobes of $|0\rangle$ point towards the (a)

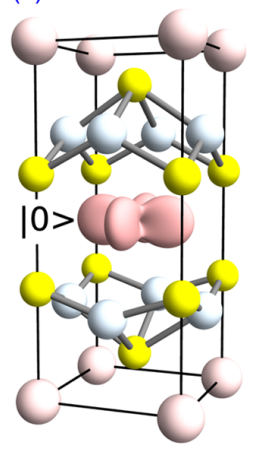

(b)

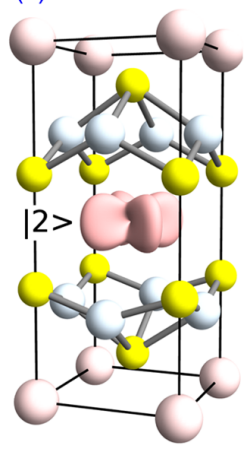

(c)

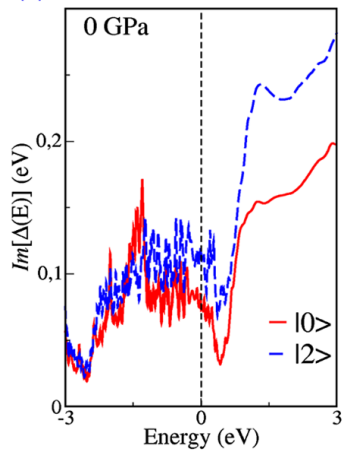

FIG. 1 (color online). (a),(b) The $\mathrm{CeCu}_{2} \mathrm{Si}_{2}$ crystal structure. The pink (large), white (medium), and yellow (small) spheres are $\mathrm{Ce}, \mathrm{Cu}$, and $\mathrm{Si}$ sites, respectively. (b) At the central Ce site, wave functions of the two CF levels are shown [ $|0\rangle$ in (a) and $|2\rangle$ in (b)]. (c) Imaginary part of the DMFT hybridization functions $\Delta$ of states $|0\rangle$ (red solid line) and $|2\rangle$ (blue dashed line) on the real energy axis at $P=0 \mathrm{GPa}$ neighboring Ce sites within the (001) plane. This difference in the spatial orientation leads to a stronger hybridization of $|2\rangle$ compared to $|0\rangle$; see Fig. 1(c). When hybridization to the itinerant bands is neglected (e.g., in the Hubbard-I approximation), the splitting of the CF levels exhibits a rather weak pressure dependence with $|0\rangle$ being the ground state, $|2\rangle$ the highest excited doublet, and the total width of about $7 \mathrm{meV}$. This "bare" CF splitting is significantly smaller than the measured one of 30-37 meV [27-29], underlining the importance of hybridization effects in this compound.

When the hybridization between $\mathrm{Ce} 4 f$ and itinerant electrons is fully included in the LDA + DMFT calculations using the CT-QMC method, the occupancies of the CF states $|0\rangle$ and $|2\rangle$ (designated by $n_{0}$ and $n_{2}$, respectively) develop a strong dependence on $P$ and $T$, which is displayed in Fig. 2 [30]. As shown there, at the highest $T=58 \mathrm{~K}$ the strongly hybridized state $|2\rangle$ dominates over the whole range of pressure. With lowering $T$ the occupancy $n_{0}$ increases for $P \lesssim 2 \mathrm{GPa}$ at the expense of $n_{2}$, while at higher $P$ the occupancies exhibit almost no temperature dependence. As a result, at the lowest temperature $T=7 \mathrm{~K}$ that we reached, the state $|0\rangle$ dominates at ambient and negative $P$ and its occupancy drops sharply between 0 and $2 \mathrm{GPa}$. In the inset of Fig. 2 we map the ratio $n_{0} / n_{2}$ as a function of $P$ and $T$. The resulting "phase diagram" can be divided into two domains: the low- $P$-low- $T$ region with the Ce $4 f$ mostly in the state $|0\rangle$

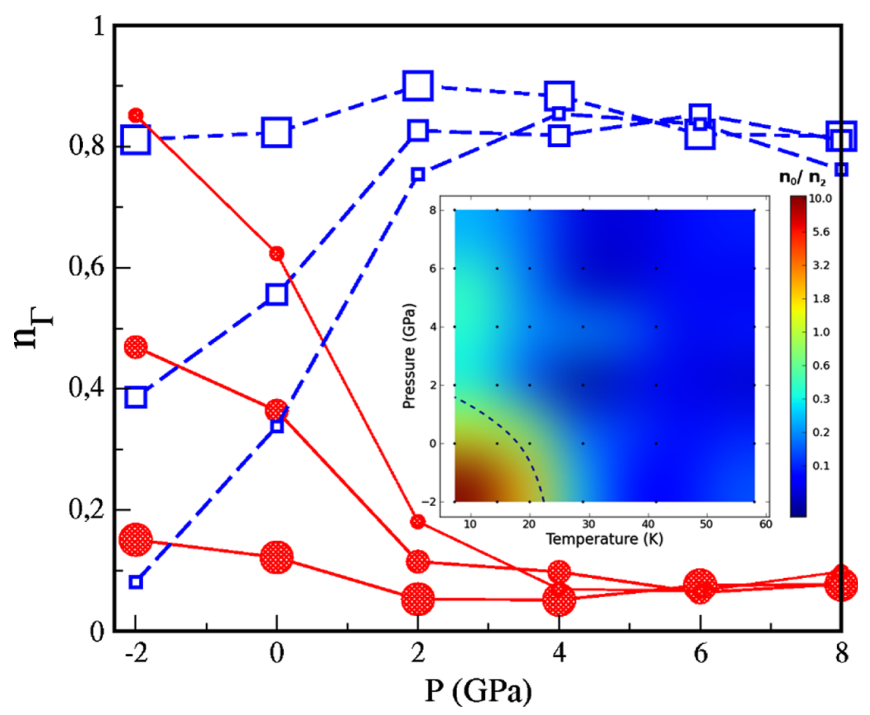

FIG. 2 (color online). Occupancies $n_{0}$ (red circles) and $n_{2}$ (blue squares) of the CF states $|0\rangle$ and $|2\rangle$, as a function of pressure and temperature. The large, medium, and small symbols denote the occupancies at 58, 14, and $7 \mathrm{~K}$, respectively. The curves are linear interpolations between the corresponding points. Inset: the $(T, P)$ map of the $n_{0} / n_{2}$ ratio. The dots indicate the values of $T$ and $P$ for which the LDA + DMFT calculations were performed. The dashed line is the $n_{0}=n_{2}$ boundary between the two regions, see text. 
(a)

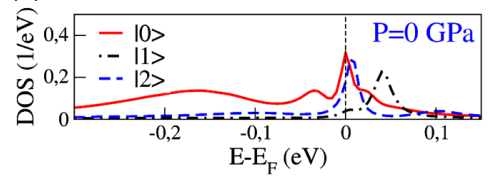

(c)

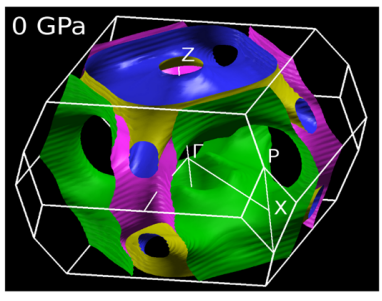

(b)

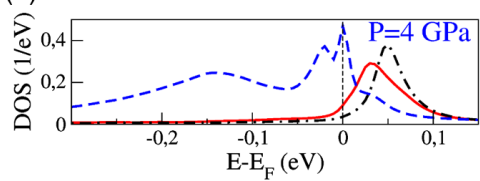

(d)

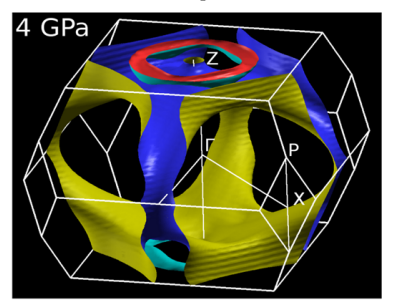

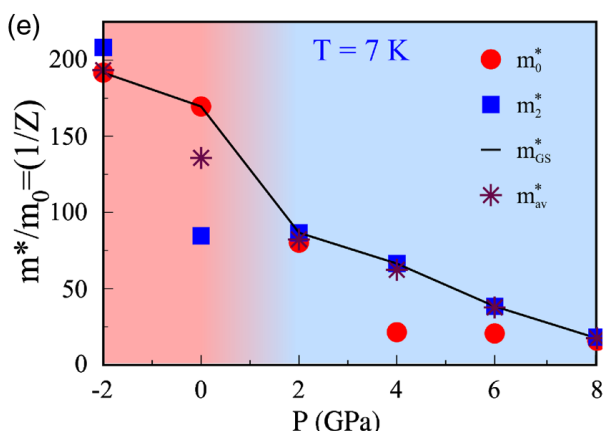

FIG. 3 (color online). (a),(b) The LDA + DMFT partial densities of states of $|0\rangle,|1\rangle$, and $|2\rangle$ states in the vicinity of the Fermi level at $P=0$ (a) and $4 \mathrm{GPa}$ (b) at $T=7 \mathrm{~K}$. The orbital character of the main Kondo peak changes as $P$ increases. (c),(d) The corresponding Fermi surfaces (FS) at the same temperature. One may notice that the large colored in green (or light gray) FS sheet present at zero pressure disappears at $4 \mathrm{GPa}$. (e) The mass enhancements $m_{\mathrm{GS}}^{*}, m_{0}$, and $m_{2}$ for the most-occupied, $|0\rangle$, and $|2\rangle$ states, respectively, as well as the average one $m_{\mathrm{av}}^{*}$ as a function of $P$ at $T=7 \mathrm{~K}$.

and the rest, where the state $|2\rangle$ dominates. The boundary $n_{0}=n_{2}$ between these two domains (dashed line in inset of Fig. 2) extrapolated to $T=0$ gives $P_{\mathrm{cr}} \approx 2.7 \mathrm{GPa}$ [31]. Recent q-dependent NIXS measurements [32] found Ce $4 f$ in the state $|2\rangle$ at ambient pressure and $T=20 \mathrm{~K}$, in agreement with our calculations. In contrast to the orbital occupancies, the total calculated occupancy of the Ce $4 f$ shell shows modest dependence on pressure

At a qualitative level, this orbital transition can be captured by a periodic Anderson model consisting of two localized levels split by a CF field $\Delta_{\mathrm{CF}}$, and such that the hybridization of the lowest level $(|0\rangle)$ with itinerant bands is (approximately twice) smaller than that of the excited level $(|2\rangle)$, as introduced in Ref. [33]. The resulting orbital occupancy versus $(V, T)$ map [34] for this model at low to moderate $T$ is remarkably similar to the one of $\mathrm{CeCu}_{2} \mathrm{Si}_{2}$ shown in Fig. 2. We note that the critical strength of hybridization $V_{\text {cr }}$ for the transition in the two-level PAM can be estimated from the condition $\Delta_{\mathrm{CF}}=T_{K \text {,ex }}-$ $T_{K, \mathrm{GS}} \approx T_{K \text {,ex }}$, where $T_{K, \text { ex (GS) }}$ is the single-impurity Kondo scale for the excited (ground-state) level and $T_{K \text {,ex }} \gg T_{K, \mathrm{GS}}$ due to exponential dependence of $T_{K}$ on the hybridization strength.

The low-energy electronic structure of $\mathrm{CeCu}_{2} \mathrm{Si}_{2}$ is also affected by the orbital transition. In Figs. 3(a) and 3(b) we display the partial densities of states [(PDOS), or orbitalresolved spectral functions] of the $|0\rangle,|1\rangle$, and $|2\rangle$ orbitals in the vicinity of the Fermi level $E_{F}$ at pressures of 0 and $4 \mathrm{GPa}$, respectively, for $T=7 \mathrm{~K}$ [35]. One sees that the Kondo peak due to the Ce $4 f$ quasiparticle states located at $E_{F}$ changes its orbital character from $|0\rangle$ to $|2\rangle$ after the system passes through the orbital transition between those two pressures. The spectral weights of the peak has also increased with $P$ due to enhancement of the Kondo scale. The occupied spin-orbit and CF peaks at ambient $P$ are located at $-0.2 \mathrm{eV}$ and $-35 \mathrm{meV}$, respectively, in agreement with recent photoemission measurements [29]. They are shifted to somewhat lower energies and change their orbital character at $P=4 \mathrm{GPa}$. The prominent CF satellite peaks above $E_{F}$ in Fig. 3 (a) are due to empty (or weakly occupied) $\mathrm{CF}$ states, their positions with respect to the Fermi level define the renormalized CF splitting. The calculated zero-pressure $\mathrm{CF}$ splitting of about $40 \mathrm{meV}$ is in agreement with the experimental value of $30-37 \mathrm{meV}$ [27-29] and exhibits a moderate increase with $P$, with the orbital character of the second CF peak switching across the transition. A similar evolution is observed as function of temperature at the ambient pressure [35]: a Kondo peak of the $|0\rangle$ character at $T=7 \mathrm{~K}$ transforms into a two-peak structure and shifted away from $E_{F}$ at higher $T$. However, in this case one sees no clear Kondo resonance of the orbital character $|2\rangle$ at $T \geq 14 \mathrm{~K}$ in agreement with experimental estimates $T_{K} \approx 10 \mathrm{~K}$ for $\mathrm{CeCu}_{2} \mathrm{Si}_{2}$ at ambient $P$ [27].

We have also calculated the corresponding Fermi surfaces (FS) for $T=7 \mathrm{~K}$, which are displayed for the same conditions of $P=0$ and $4 \mathrm{GPa}$ in Figs. 3(c) and 3(d), respectively [36]. One sees a very clear impact of the orbital transition on the FS topology: a large FS sheet present at ambient pressure (i.e., in the domain of state $|0\rangle$ ) disappears completely when the system passes to the domain of state $|2\rangle$, while another sheet is significantly deformed.

We extracted the orbital-resolved mass enhancements $m_{\Gamma}^{*}$ from the corresponding self-energies $\Sigma_{\Gamma \Gamma}(i \omega)$ on the Matsubara grid as $1-\left[d \operatorname{Im} \Sigma_{\Gamma \Gamma}(i \omega) /\left.d \omega\right|_{\omega \rightarrow 0}\right]$, then the average mass enhancement $m_{\mathrm{av}}^{*}$ was computed as $\sum_{\Gamma} m_{\Gamma}^{*} N_{\Gamma}\left(E_{F}\right) / \sum_{\Gamma} N_{\Gamma}\left(E_{F}\right)$, where $N_{\Gamma}\left(E_{F}\right)$ is the corresponding PDOS at the Fermi level. As shown on Fig. 3(c), the orbital transition $|0\rangle \rightarrow|2\rangle$ is accompanied by a significant reduction of the mass enhancement $m_{\mathrm{GS}}^{*}$ of the most occupied orbital (with $|\mathrm{GS}\rangle=|0\rangle$ for $P \leq 0 \mathrm{GPa}$ and $=|2\rangle$ for $P \geq 2 \mathrm{GPa}$ ), while $m_{\mathrm{GS}}^{*}$ exhibits a rather slow linear decay away from the transition region. The evolution of the average mass enhancement $m_{\mathrm{av}}^{*}$ is generally the same as that of $m_{\mathrm{GS}}^{*}$, apart from $P=0 \mathrm{GPa}$, where the system at 
$T=7 \mathrm{~K}$ seems to be still in an intermediate state and is expected to move to the $|0\rangle$-dominated phase at lower $T$ 's (see inset in Fig. 2). This calculated mass enhancement versus $P$ evolution is in good agreement with experiment [13]. Hence, one may conclude that the system in a "heavy" Fermi-liquid state at low $P$ transforms into a "lighter" Fermi liquid through the orbital transition, at which the dominating CF state changes.

Finally, we discuss spectroscopic signatures of the orbital transition, providing a direct experimental test of our theoretical predictions in future experiments. In the past years it has been established that linear dichroism at the cerium $M$ edge of XAS (dipole transitions from $3 d$ core to $4 f$ valence states) $[37,38]$ or, even richer in information, $\mathbf{q}$ dependence (momentum transfer) in NIXS (dipole, octopole, and triakontadipole from $4 d$ core to $4 f$ valence states) $[32,39]$ directly reflect the symmetry of the local Ce wave function.

Hence, using full multiplet cluster calculations, we have simulated XAS and NIXS signals following from our theory [40]. While the XAS spectra can only probe the ground state composition by means of absolute contributions of $\mid J_{z}=5 / 2>$ and $\mid J_{z}=3 / 2>$, respectively, NIXS is capable of probing also their relative sign, i.e., the orientation of the wave function in the $a b$ plane, and, hence, distinguish states $|0\rangle$ and $|2\rangle$. We thus refer to the Supplemental Material [41] for the XAS spectra and focus here on the more informative momentum-transfer dependent NIXS signal which we report in Fig. 4. The upper panel displays, at $P=0$ and $T=7 \mathrm{~K}$, the spectral function for two different directions of momentum transfer (solid line, $\mathbf{q} \|[001]$, dashed line, $\mathbf{q} \|[100])$ at a fixed absolute value of $|\mathbf{q}|=9.3 \AA^{-1}$. Also plotted in red (dark gray) is the difference spectrum $A(\omega)_{\mathbf{q} \|[001]}-A(\omega)_{\mathbf{q} \|[100]}$. The central panel displays the evolution of this difference spectrum at fixed temperature $(7 \mathrm{~K})$ upon increasing pressure from 0 to $6 \mathrm{GPa}$. While at $0 \mathrm{GPa}$ the ground state is dominated by state $\mid 0>$ (red spectrum) the switch to a state $2>$-dominated ground state (light blue spectrum) already at $2 \mathrm{GPa}$ is clearly visible, e.g., in the respective amplitude of the first two peaks between 3 and $5 \mathrm{eV}$, or in the increase between 7 and $10 \mathrm{eV}$. We find the same clear-cut fingerprint of the orbital transition for the evolution at ambient pressure upon increasing temperature to $\approx 50 \mathrm{~K}$ (bottom panel). Also, here the change of the ground state wave function is signaled by a change of the difference spectrum. While this change is qualitatively similar to the evolution with pressure, the absolute spectra differ due to the (slightly) different absolute values of the $\mid J_{z}=5 / 2>$ and $\mid J_{z}=3 / 2>$ coefficients.

In conclusion, our first-principles LDA + DMFT calculations predict the existence of a pressure- and temperatureinduced orbital transition in $\mathrm{CeCu}_{2} \mathrm{Si}_{2}$. At this transition, the $4 f$ electron weight is transferred from CF state $|0\rangle$ (the atomic ground state for vanishing hybridization) to the excited level $|2\rangle$, because the latter hybridizes more

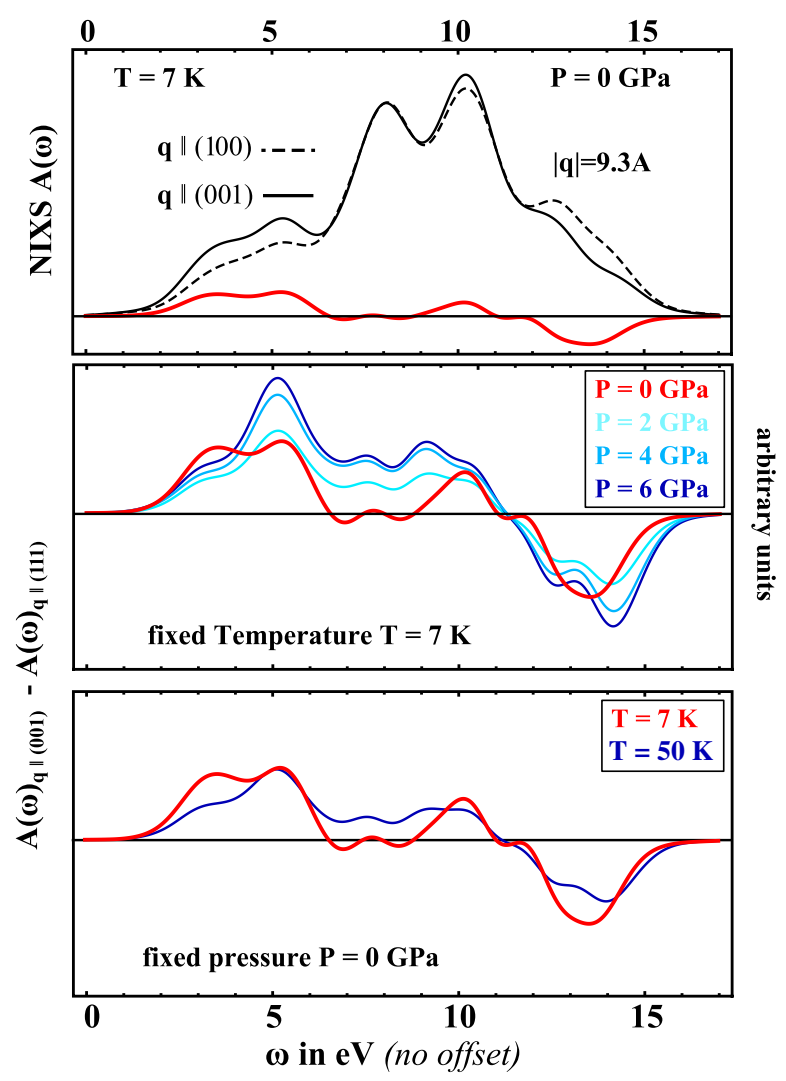

FIG. 4 (color online). Simulation of NIXS cross sections. In the top panel we show the spectral functions of the momentumtransfer-dependent spectra (solid and dashed thin black line) and the difference sprectrum (thick red line). In the middle and bottom panels we display the NIXS difference spectrum upon increasing pressure at constant $T=7 \mathrm{~K}$ and increasing temperature at ambient pressure, respectively. One may notice that the NIXS difference sprectrum undergoes structural changes due to the predicted orbital transition.

strongly with conduction electrons. Our results lead to clear-cut predictions for spectroscopic experiments like NIXS, where the fingerprint of the orbital transition can be detected in momentum-transfer dependent scattering cross sections. A similar "metaorbital" transition between two CF levels with different hybridizations has been recently discussed in the context of a two-band periodical Anderson model [33]. However, it has not been, to our knowledge, demonstrated from $a b$ initio simulations of any real heavyfermion material. It is tempting to speculate that the critical fluctuations associated with this orbital transition are responsible for the pairing in the high-pressure SC dome of $\mathrm{CeCu}_{2} \mathrm{Si}_{2}$ and isoelectronic $\mathrm{CeCu}_{2} \mathrm{Ge}_{2}$. Indeed, the calculated critical pressure of $2.5 \mathrm{GPa}$ at zero temperature is rather close to the experimental maximum of this dome. Similar orbital transitions between CF levels may also explain the superconductivity away from magnetic quantum points in other HF compounds. It will be interesting to investigate whether such orbital transitions are always related to a double-dome SC or whether they can as well 
occur in single-dome and non-SC HF compounds (e.g., $\mathrm{CePd}_{2} \mathrm{Si}_{2}, \mathrm{CeAl}_{2}$ ). We finally note that "composite pairing" SC has been proposed theoretically [42] to arise at the boundary between two distinct HF liquids originating in two orthogonal CF levels.

We acknowledge discussions with D. Jaccard, who attracted our attention to this problem, as well as with J.-P. Rueff, T. Willers, A. Severing, and M. Haverkort. Computing resources were provided by the Swedish National Infrastructure for Computing (SNIC) at the National Supercomputer Centre (NSC) and PDC Center for High Performance Computing, IDRIS-GENCI, and the Swiss Center for Scientific Computing.

[1] F. Steglich, J. Aarts, C. D. Bredl, W. Lieke, D. Meschede, W. Franz, and H. Schäfer, Phys. Rev. Lett. 43, 1892 (1979).

[2] B. Bellarbi, A. Benoit, D. Jaccard, J. M. Mignot, and H. F. Braun, Phys. Rev. B 30, 1182 (1984).

[3] F. Thomas, J. Thomasson, C. Ayache, C. Geibel, and F. Steglich, Physica (Amsterdam) 186B-188B, 303 (1993).

[4] G. Seyfarth, A.-S. Rüetschi, K. Sengupta, A. Georges, and D. Jaccard, Europhys. Lett. 98, 17012 (2012).

[5] G. Seyfarth, A.-S. Rüetschi, K. Sengupta, A. Georges, D. Jaccard, S. Watanabe, and K. Miyake, Phys. Rev. B 85, 205105 (2012)

[6] E. Vargoz and D. Jaccard, J. Magn. Magn. Mater. 177-181, 294 (1998).

[7] F. Grosche, S. Julian, N. Mathur, and G. Lonzarich, Physica (Amsterdam) 223B-224B, 50 (1996).

[8] R. Movshovich, T. Graf, D. Mandrus, J. D. Thompson, J. L. Smith, and Z. Fisk, Phys. Rev. B 53, 8241 (1996).

[9] H. Q. Yuan, F. M. Grosche, M. Deppe, C. Geibel, G. Sparn, and F. Steglich, Science 302, 2104 (2003).

[10] E. Lengyel, M. Nicklas, H. S. Jeevan, C. Geibel, and F. Steglich, Phys. Rev. Lett. 107, 057001 (2011).

[11] F. Steglich et al., Physica (Amsterdam) 223B-224B, 1 (1996).

[12] O. Stockert et al., Nat. Phys. 7, 119 (2011).

[13] A. T. Holmes, D. Jaccard, and K. Miyake, J. Phys. Soc. Jpn. 76, 051002 (2007).

[14] D. Jaccard and A. T. Holmes, Physica (Amsterdam) 359B361B, 333 (2005).

[15] K. Miyake, J. Phys. Condens. Matter 19, 125201 (2007).

[16] Y. Onishi and K. Miyake, J. Phys. Soc. Jpn. 69, 3955 (2000).

[17] J.-P. Rueff, S. Raymond, M. Taguchi, M. Sikora, J.-P. Itié, F. Baudelet, D. Braithwaite, G. Knebel, and D. Jaccard, Phys. Rev. Lett. 106, 186405 (2011).

[18] M. Aichhorn, L. Pourovskii, V. Vildosola, M. Ferrero, O. Parcollet, T. Miyake, A. Georges, and S. Biermann, Phys. Rev. B 80, 085101 (2009).

[19] M. Aichhorn, L. Pourovskii, and A. Georges, Phys. Rev. B 84, 054529 (2011).

[20] P. Blaha, K. Schwarz, G. Madsen, D. Kvasnicka, and J. Luitz, WIEN2k, An Augmented Plane Wave + Local Orbitals Program for Calculating Crystal Properties
(Techn. Universitat Wien, Austria, 2001), ISBN 3-9501031-1-2.

[21] A. Georges, G. Kotliar, W. Krauth, and M. J. Rozenberg, Rev. Mod. Phys. 68, 13 (1996).

[22] E. Gull, A. J. Millis, A. I. Lichtenstein, A. N. Rubtsov, M. Troyer, and P. Werner, Rev. Mod. Phys. 83, 349 (2011).

[23] M. Ferrero and O. Parcollet, TRIQS: A Toolbox for Research on Interacting Quantum Systems, http://ipht.cea .fr/triqs.

[24] See Supplemental Material at http://link.aps.org/ supplemental/10.1103/PhysRevLett.112.106407 for the calculational details.

[25] I. Spain, F. Steglich, U. Rauchschwalbe, and H. Hochheimer, Physica B+C (Amsterdam) 139-140, 449 (1986), ISSN 0378-4363.

[26] The negative-pressure lattice parameters have been obtained by extrapolating data from Ref. [25].

[27] S. Horn, E. Holland-Moritz, M. Loewenhaupt, F. Steglich, H. Scheuer, A. Benoit, and J. Flouquet, Phys. Rev. B 23, 3171 (1981).

[28] E. A. Goremychkin and R. Osborn, Phys. Rev. B 47, 14280 (1993).

[29] D. Ehm, S. Hüfner, F. Reinert, J. Kroha, P. Wölfle, O. Stockert, C. Geibel, and H. v. Löhneysen, Phys. Rev. B 76, 045117 (2007).

[30] The occupancy of the state $|1\rangle$ remains small $\left(n_{1} \approx 0.1\right)$ over the whole range of $P$ and $T$.

[31] See Sec. III of Supplemental Material at http://link.aps.org/ supplemental/10.1103/PhysRevLett.112.106407 for details of the extrapolation to $T=0$.

[32] T. Willers et al., Phys. Rev. Lett. 109, 046401 (2012).

[33] K. Hattori, J. Phys. Soc. Jpn. 79, 114717 (2010).

[34] See Supplemental Material at http://link.aps.org/ supplemental/10.1103/PhysRevLett.112.106407 for the periodic Anderson model calculations and the resulting orbital occupancy versus $(V, T)$ map.

[35] See Sec. IV of Supplemental Material at http://link.aps.org/ supplemental/10.1103/PhysRevLett.112.106407 for a detailed pressure and temperature evolution of partial DOS.

[36] See Sec. V of Supplemental Material at http://link.aps.org/ supplemental/10.1103/PhysRevLett.112.106407 for plots of Fermi surfaces obtained for the range of pressures from -2 to $6 \mathrm{GPa}$.

[37] P. Hansmann et al., Phys. Rev. Lett. 100, 066405 (2008).

[38] T. Willers et al., Phys. Rev. B 85, 035117 (2012).

[39] M. W. Haverkort, A. Tanaka, L. H. Tjeng, and G. A. Sawatzky, Phys. Rev. Lett. 99, 257401 (2007).

[40] For the simulations we used the code of Ref. [43] and parameters for the Slater integrals and the spin-orbit coupling is the same as in [37]. For the NIXS spectra, the radial part of the cross section was taken from [38].

[41] See Sec. VI of Supplemental Material at http://link.aps.org/ supplemental/10.1103/PhysRevLett.112.106407 for XAS and NIXS spectra calculated for the whole temperaturepressure range under consideration.

[42] R. Flint, M. Dzero, and P. Coleman, Nat. Phys. 4, 643 (2008).

[43] M. W. Haverkort, M. Zwierzycki, and O. K. Andersen, Phys. Rev. B 85, 165113 (2012). 